\title{
Fully Digital Transmit Equalizer With Dynamic Impedance Modulation
}

\author{
Ranko Sredojević, Student Member, IEEE, and Vladimir Stojanović, Member, IEEE
}

\begin{abstract}
This paper analyzes the energy efficiency of different transmit equalizer driver topologies. Dynamic impedance modulation is found to be the most energy-efficient mechanism for transmit pre-emphasis, when compared with impedance-maintaining current and voltage-mode drivers.

The equalizing transmitter is implemented as a digital push-pull impedance-modulating (RM) driver with fully digital RAM-DAC back-end for pattern lookup. This back-end compensates for both duty-cycle distortion and driver nonlinearity, while providing a programmable pre-emphasis. A testchip fabricated in 90-nm CMOS process shows relatively small signal degradation from dynamic modulation of driver output impedance over a variety of $20^{\prime \prime}$ backplanes at $4 \mathrm{~Gb} / \mathrm{s}$, with energy efficiency of $2 \mathrm{pJ} / \mathrm{bit}$ at $100 \mathrm{mV}$ of receiver eye. Despite this signal degradation, at the same performance point, the impedance modulating driver shows better energy efficiency than impedance-maintaining current and voltage-mode drivers.
\end{abstract}

Index Terms-Channel impedance matching, high-speed link, transmit equalization.

\section{INTRODUCTION}

$\mathbf{S}$ CALING of system throughputs and memory bandwidth requirements has increased the focus on energy efficiency of high-speed link and I/O circuits in power-limited VLSI systems. In today's large systems-on-a-chip, communication infrastructure including high-speed I/Os takes up a significant portion of power, limiting the amount left for useful computation [1].

The conflicting bandwith and power scaling requirements have stimulated vigorous research activities resulting in significant improvements in link energy efficiency [2]-[6], [11]. These improvements in energy efficiency have focused on most dominant subsystems, such as clocking and signaling transmit-receive chain. To that end, voltage-mode (VM) drivers have been introduced instead of current-mode (CM) drivers to improve the energy efficiency of the transmitter [2], [3]. However, these VM drivers suffer from a power penalty when used to implement a transmit pre-emphasis filter [2], which is particularly well suited for asymmetric-complexity link channel applications such as memory interfaces [4] and lossy channels with long intersymbol interference (ISI) tails such as cables or silicon carriers [5], [11].

Both CM and VM transmitters incur significant power penalty when configured to provide a matched, low-impedance termination to the channel over a wide range of output voltages necessary for pre-emphasis. However, this matching only notionally improves the performance of the link, since it only minimizes the unequalized ISI. In practice, trace fabrication tolerances affect the variation of the channel characteristic impedance. On-die and packaging parasitic capacitances at the transmitter and receiver, as well as process variations in the driver output stage, can also significantly alter the actual termination impedance and matching to the channel. Hence, the blind sacrifice of power efficiency in order to achieve the nominal impedance match should be reevaluated in the context of true link performance metrics. To do this, we must first better understand the tradeoff between signal degradation due to transmitter impedance mismatch and power efficiency improvement.

In our analysis, we show that power-efficiency improvements over the VM transmit-equalization scheme must come from the controlled relaxation of impedance matching constraints on common-mode and/or differential-mode matching. One design that makes such a tradeoff, having frequency-selective common-mode matching for improved power efficiency, is given in [6].

In this study, we take a step further in improving the power efficiency, by reexamining the benefits of the static differential impedance matching and analyzing the possible tradeoffs if this constraint is removed. To facilitate the analysis, we first introduce appropriate metrics for energy-efficiency comparison of transmit equalizers and use them to show that most efficient driver topology is based on dynamic resistance-modulation (RM) of transmitter impedance, which we first presented in [7]. The remaining sections discuss the design and experimental results of the proposed RM pre-emphasis transmitter concept, which achieves record-high energy efficiency at same performance levels as transmit equalizers using CM and VM drivers.

\section{Power-Efficiency Metrics for LinK Transmitters}

Manuscript received December 04, 2010; revised February 05, 2011; accepted April 01, 2011. Date of publication June 09, 2011; date of current version July 22, 2011. This paper was approved by Guest Editor Pavan Kumar Hanumolu. This work was supported by the C2S2 Focus Center, one of six research centers founded under the Focus Center Research Program (FCRP), a Semiconductor Research Corporation entity.

The authors are with the Massachusetts Institute of Technology, Cambridge, MA 02139 USA (e-mail: rasha@mit.edu).

Color versions of one or more of the figures in this paper are available online at http://ieeexplore.ieee.org.

Digital Object Identifier 10.1109/JSSC.2011.2151530
Here, we analyze the equalization mechanisms and power efficiency of different transmitter driver topologies. The main objective of a link transmit-receive signaling subsystem is to neutralize the ISI resulting from the signal dispersion and reflections in the communication channel. Thus, the communication channel completely determines the appropriate transmitter and receiver signal processing actions. 
Most often, transmit equalization is done by finite-impulseresponse (FIR) filters designed to reduce, or completely eliminate, the equalized impulse response ISI. Assuming that the desired transmitter impulse response is given by a vector $\boldsymbol{a}$, it is possible to calculate all of the encoded voltages that such an FIR transmitter would produce at its output. We convolve all of the possible filter states (data patterns) with its impulse response $\boldsymbol{a}$ to calculating the output values

$$
v_{b_{0} \ldots b_{k-1}}=\sum a_{i} b_{k-i-1}
$$

where each $b_{0} \ldots b_{k-1} \in\{-1,1\}^{k}$ is one possible filter state (data symbol sequence) and $v_{b_{0} \ldots b_{k-1}}$ is the appropriate encoded voltage at its output.

Given a specific transmit-driver topology, every voltage at the transmitter's output can be associated with a certain power cost necessary to maintain it. Thus, each transmitter topology can be described by the power consumed to achieve certain output voltage, i.e., its static power characteristic $P_{\text {out }}\left(V_{\text {out }}\right)$.

When coupled to a specific channel, we should consider the average power necessary to produce the set of encoded voltages to equalize the channel. For example, in the case of an uncoded, uniformly distributed incoming symbol sequence, all of the sequences are equally likely, and we should minimize

$$
P=\frac{1}{2^{k}} \sum_{b_{0} \ldots b_{k-1}} P_{\text {out }}\left(v_{b_{0} \ldots b_{k-1}}\right)
$$

or a similar expression depending on the appropriate probability of each encoded output voltage.

The performance analysis of driver topologies is based on this average power metric (2), and we derive it first for $\mathrm{CM}$ and VM transmit-equalizers. For fair comparison of CM- and VM-type drivers, we assume that the maximum output signal (sometimes referred to as the peak power constraint or peak voltage constraint) from the transmitter is $V_{\mathrm{DD}} / 2$. The analysis is performed for the static (dc) case, but is also valid if the receiver termination is matched to the channel impedance, as, in that case, the channel and the receiver can be replaced with lumped impedance at the transmitter's output.

In Fig. 1, we show a segmented CM driver implementing a two-tap FIR filter. ${ }^{1}$ Usually, the transmit and receive sides of the channel are connected to the supply through the channel terminating resistors to maintain both the differential and $\mathrm{CM}$ channel impedance match. The configuration in Fig. 1 is equivalent to the standard case in terms of power consumption for a given maximum output voltage, but only provides differential impedance match at the receiver. The control signals $\mathrm{B}[0]$ and $\mathrm{B}[-1]$ denote the current and the previous bit (symbol) and $\mathrm{Bn}[]$ appropriate bit inversions.

From the Norton equivalent in Fig. 1(b), we can write

$$
v_{\text {out }}=\left(R_{\mathrm{tx}} \| R_{\mathrm{rx}}\right) \Delta i
$$

${ }^{1}$ In this study, following the practice in the circuit-design community, we will use the word "tap" to designate the number of coefficients in a filter polynomial. Alternatively, we could express this in terms of the "order" of the filter polynomial, which is the minimal number of the delay elements needed to implement a filter. For example, a two-tap FIR filter implements a first-order transfer function.

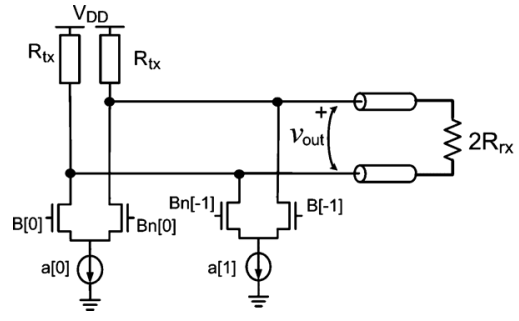

(a)

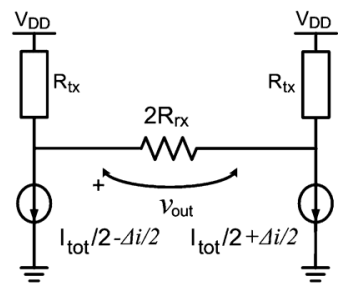

(b)
Fig. 1. (a) Tap-segmented $\mathrm{CM}$ transmit equalizer and (b) $\mathrm{CM}$ equivalent schematic.

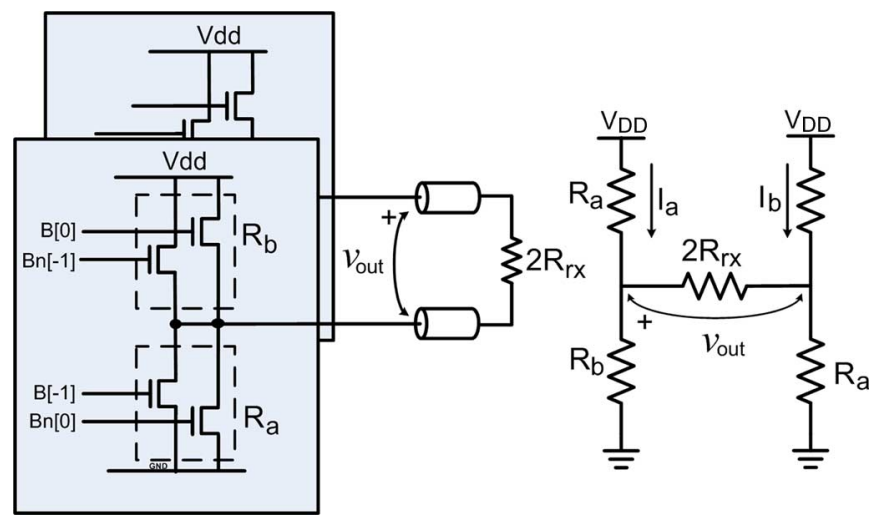

Fig. 2. (a) Tap-segmented VM transmit equalizer operates as a programmable voltage divider. (b) VM equivalent schematic.

where $\Delta i$ is the total differential current of the switches, and transmitter and receiver single-ended terminations are $R_{t x}$ and $R_{r x}$, respectively. In the extreme case when maximum output signal is being produced, (3a) becomes

$$
V_{\mathrm{DD}} / 2=\left(R_{t x} \| R_{r x}\right) I_{\mathrm{tot}}
$$

where $I_{\text {tot }}$ is the sum of all of the tail currents. As CM drivers always consume the same amount of current, we can write

$$
P_{\text {out }}\left(v_{\text {out }}\right)=V_{\mathrm{DD}} I_{\text {tot }}=\frac{V_{\mathrm{DD}}^{2}}{2\left(R_{\mathrm{tx}} \| R_{\mathrm{rx}}\right)}
$$

which, in the fully matched case [2], [3], [6] of $R_{\mathrm{tx}}=R_{\mathrm{rx}}=$ $50 \Omega$, becomes

$$
P_{\text {out }}\left(v_{\text {out }}\right)=2\left(\frac{V_{\text {DD }}^{2}}{100 \Omega}\right) .
$$

Two main problems of the CM topology are the high voltage headroom needed to keep transistors in the proper region of operation and the implementation of the matching network. The matching resistors in the $\mathrm{CM}$ topology are connected in parallel with the channel, thus creating a current divider. In the matched impedance case, (3d), only half of the transmitter current is sent into the channel regardless of the output voltage, significantly degrading the power efficiency.

This motivated the use of VM-type drivers, shown in Fig. 2(a). When not equalizing, VM driver's termination network appears in series with the receiver, thus limiting the maximum output voltage to $V_{\mathrm{DD}} / 2$. No parallel shunts exist in this configuration, and the driver is very efficient. However, 


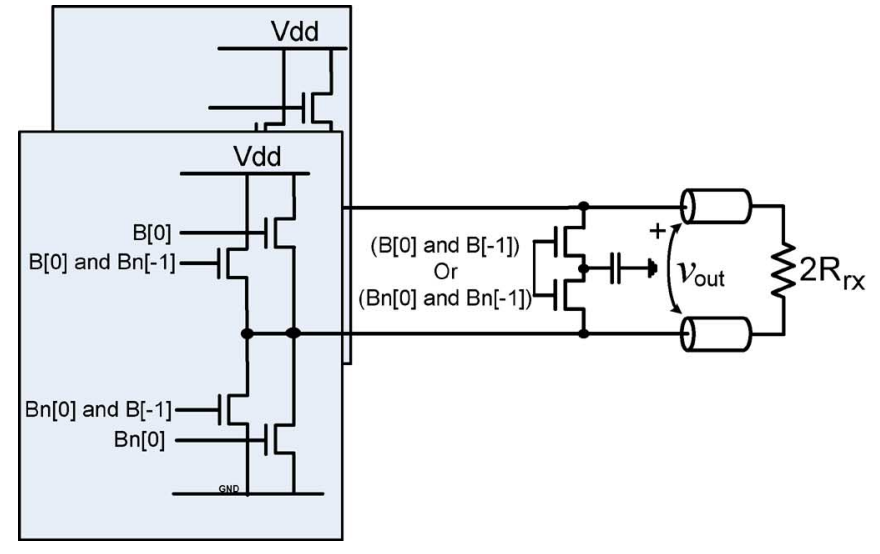

(a)

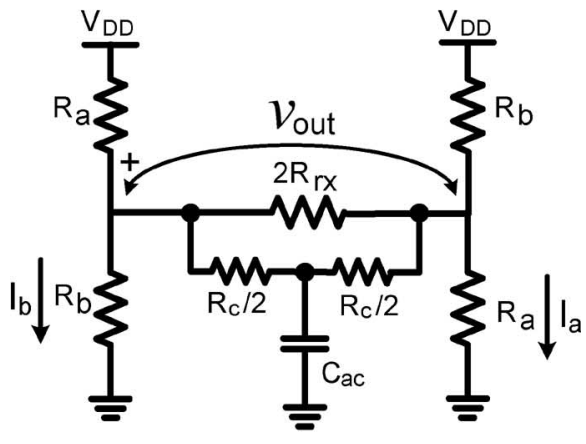

(b)

Fig. 3. (a) Tap-segmented $\mathrm{VM}^{*}$ equalizer driver performs partial impedance matching in the driver itself and accurate match by complementing the driver with the full differential and partial CM matching network. (b) $\mathrm{VM}^{*}$ equivalent schematic.

when used as equalizers, VM drivers operate by creating different voltage dividers between the output driver segments that correspond to tap weights (Fig. 2). This leads to current shunts in parallel with the channel, and the power efficiency [see (2)] degrades.

Assuming symmetric switching of the transmitter $\mathrm{P}$ and $\mathrm{N}$ sides, we can write

$$
P_{\mathrm{out}}=V_{\mathrm{DD}}\left(I_{a}+I_{b}\right)
$$

where $I_{a}$ and $I_{b}$ are currents drawn from the supply through the two branches (tap segments) of the transmitter output stage [Fig. 2(b)]. By observing the anti-symmetry in the H-bridge of the VM driver topology and denoting by $R_{a}$ and $R_{b}$ the appropriate pull-up/pull-down branches of the $\mathrm{N}$ and $\mathrm{P}$ sides of the driver as in Fig. 2(a), it follows that

$$
\begin{aligned}
& I_{a}=\frac{V_{\mathrm{DD}}-\left(v_{\mathrm{out}} / 2+V_{\mathrm{DD}} / 2\right)}{R_{a}} \\
& I_{b}=\frac{V_{\mathrm{DD}}-\left(-v_{\mathrm{out}} / 2+V_{\mathrm{DD}} / 2\right)}{R_{b}} .
\end{aligned}
$$

Substituting (4b) and (4c) into (4a) and observing that $R_{\mathrm{tx}}=$ $R_{a} \| R_{b}$ gives after factoring

$$
P_{\text {out }}\left(v_{\text {out }}\right)=\frac{V_{\mathrm{DD}}^{2}}{2 R_{\mathrm{tx}}}\left(1+\frac{v_{\text {out }}}{V_{\mathrm{DD}}} R_{\mathrm{tx}}\left(\frac{1}{R_{b}}-\frac{1}{R_{a}}\right)\right) .
$$

Applying Thevenin's theorem to N/P side of the transmit driver [Fig. 2(b)] allows us to express $v_{\text {out }}$ as

$$
v_{\text {out }}=\left(\frac{R_{b}}{R_{a}+R_{b}} V_{\mathrm{DD}}-\frac{R_{a}}{R_{a}+R_{b}} V_{\mathrm{DD}}\right) \frac{2 R_{\mathrm{rx}}}{2 R_{\mathrm{rx}}+2 R_{\mathrm{tx}}}
$$

and after factoring

$$
v_{\text {out }}=R_{\mathrm{tx}} V_{\mathrm{DD}}\left(\frac{1}{R_{b}}-\frac{1}{R_{a}}\right) \frac{2 R_{\mathrm{rx}}}{2 R_{r x}+2 R_{\mathrm{tx}}} .
$$

Finally, combining (4d) and (4f), we see that

$$
P_{\text {out }}\left(v_{\text {out }}\right)=\left(\frac{V_{\mathrm{DD}}^{2}}{2 R_{\mathrm{tx}}}\right)\left(1-\left(1+\frac{R_{\mathrm{tx}}}{R_{\mathrm{rx}}}\right)\left(\frac{v_{\text {out }}}{V_{\mathrm{DD}}}\right)^{2}\right)
$$

provided that $v_{\text {out }}$ is in the feasible range of the driver. In the fully matched case of $R_{\mathrm{tx}}=R_{\mathrm{rx}}=50 \Omega$, we can evaluate ( $\left.4 \mathrm{~g}\right)$ to

$$
P_{\text {out }}\left(v_{\text {out }}\right)=\left(\frac{V_{\mathrm{DD}}^{2}}{100 \Omega}\right)\left(1-2\left(\frac{v_{\text {out }}}{V_{\mathrm{DD}}}\right)^{2}\right)
$$

which explains the power increase when small differential voltages are produced. This makes it more desirable to operate VM drivers in series-terminated mode without any equalization [3], whenever possible.

An effective redesign of the VM driver, presented in [6], is shown in Fig. 3, labeled here as $\mathrm{VM}^{*}$ driver. This driver reduces the amount of crowbar current produced by introducing additional termination switches between the channel input ports [Fig. 3(a)]. In this configuration, impedance matching is done by the driver only in part and supplemented by the differential termination switches. It is obvious that, when zero output voltage is transmitted, there is no current delivered into the channel, but $\mathrm{VM}^{*}$ consumes the same amount of power as for the maximum signal being sent. This indicates that the shunting still exists in this driver, but at a significantly reduced level. The role of the shunt current is to maintain constant supply current, which is desirable in the absence of the supply regulator.

Simple analysis corroborated by claims in [6] shows that

$$
P_{\text {out }}\left(V_{\text {out }}\right) \approx \frac{1}{2}\left(\frac{V_{\text {DD }}^{2}}{100 \Omega}\right)
$$

for $100-\Omega$ differential termination match. To see this, we can analyze the equivalent circuit shown in Fig. 3(b).

The output voltage of the $\mathrm{VM}^{*}$ driver can be expressed as

$$
V_{\text {out }}=\frac{R_{b}-R_{a}}{R_{b}+R_{a}} V_{\mathrm{DD}} \frac{R_{c} \|\left(2 R_{\mathrm{rx}}\right)}{2\left(R_{a} \| R_{b}\right)+R_{c} \|\left(2 R_{\mathrm{rx}}\right)}
$$

or

$$
V_{\text {out }}=V_{\mathrm{DD}} \frac{R_{b} R_{c}-R_{a} R_{c}}{2 \frac{R_{a} R_{b} R_{c}}{\left(2 R_{r x}\right)}+2 R_{a} R_{b}+R_{a} R_{c}+R_{b} R_{c}}
$$


after the substitution of the "||" operator. Furthermore, the termination constraint, to be analyzed in more detail later in the text, can be written as

$$
R_{a}\left\|R_{b}\right\| \frac{R_{c}}{2}=R_{m}
$$

where $R_{m}$ is some fixed resistance, usually equal to the characteristic impedance of the channel. In expanded form it can be written as

$$
\frac{R_{a} R_{b} R_{c}}{2 R_{a} R_{b}+R_{a} R_{c}+R_{b} R_{c}}=R_{m}
$$

Finally, the supply current can be expressed as

$$
\left(\frac{V_{\mathrm{DD}}}{2}+\frac{V_{\mathrm{out}}}{2}\right) \frac{1}{R_{b}}+\left(\frac{V_{\mathrm{DD}}}{2}-\frac{V_{\mathrm{out}}}{2}\right) \frac{1}{R_{a}}=I_{\mathrm{DD}} .
$$

From (5c) and (5e), we can write

$$
V_{\text {out }}=V_{\mathrm{DD}} \frac{R_{b}-R_{a}}{2 \frac{R_{a} R_{b}}{\left(2 R_{r x}\right)}+\frac{R_{a} R_{b}}{R_{m}}}
$$

which enables us to find the relationship between $R_{b}$ and $R_{a}$ as

$$
R_{a}=\frac{R_{b}}{1+k R_{b}},
$$

where the coefficient $k$ is given by

$$
k=\frac{V_{\mathrm{out}}}{V_{\mathrm{DD}}}\left(\frac{2}{\left(2 R_{\mathrm{rx}}\right)}+\frac{1}{R_{m}}\right)=q V_{\mathrm{out}}
$$

and we define the coefficient $q$ in order to achieve a form explicit in terms of $V_{\text {out }}$ in order to facilitate the final analysis.

After substituting the last equation into (5f), we get the relationship between $V_{\text {out }}, I_{\mathrm{DD}}$ and $R_{b}$ as

or

$$
\frac{V_{\mathrm{DD}}}{2} \frac{2+k R_{b}}{R_{b}}-k \frac{V_{\mathrm{out}}}{2}=I_{\mathrm{DD}}
$$

$$
\frac{V_{\mathrm{DD}}}{R_{b}}+q \frac{V_{\mathrm{DD}}}{2} V_{\text {out }}-q \frac{V_{\mathrm{out}}^{2}}{2}=I_{\mathrm{DD}}
$$

explicitly in terms of $V_{\text {out }}$. To ensure the constant supply current operation, we should have

$$
\frac{d I_{\mathrm{DD}}}{d V_{\text {out }}}=0
$$

resulting in the differential equation that defines $R_{b}$ as

$$
\frac{d R_{b}}{d V_{\text {out }}}=q R_{b}^{2}\left(\frac{1}{2}-\frac{V_{\text {out }}}{V_{\mathrm{DD}}}\right) .
$$

Resolving this results in the general form of $R_{b}\left(V_{\text {out }}\right)$ dependence

$$
R_{b}=\frac{1}{\frac{1}{R_{\mathrm{bmin}}}-\frac{q}{2}\left(V_{\mathrm{out}}+\frac{V_{\mathrm{DD}}}{2}-\frac{1}{V_{\mathrm{DD}}}\left(V_{\mathrm{out}}^{2}-\left(\frac{V_{\mathrm{DD}}}{2}\right)^{2}\right)\right)}
$$

where the coefficient $q$ was defined in (5i). Once the $R_{b}\left(V_{\text {out }}\right)$ is known, we can determine $R_{a}=R_{b}\left(-V_{\text {out }}\right)$. Finally, for $R_{\mathrm{bmin}}=R_{m}=R_{\mathrm{rx}}=50 \Omega,-0.5 V_{\mathrm{DD}} \leq V_{\text {out }} \leq 0.5 V_{\mathrm{DD}}$ and $I_{\mathrm{DD}}=(1 / 2)\left(V_{\mathrm{DD}} / 100 \Omega\right)$, we can check that $R_{c}$, calculated from (5d) after $R_{a}$ and $R_{b}$ are known, is nonnegative, and thus well defined.

We should note that, in Figs. 1-3, for the purpose of deriving the appropriate equivalent models, we have shown the tap-segmented representations of the $\mathrm{CM}, \mathrm{VM}$, and $\mathrm{VM}^{*}$

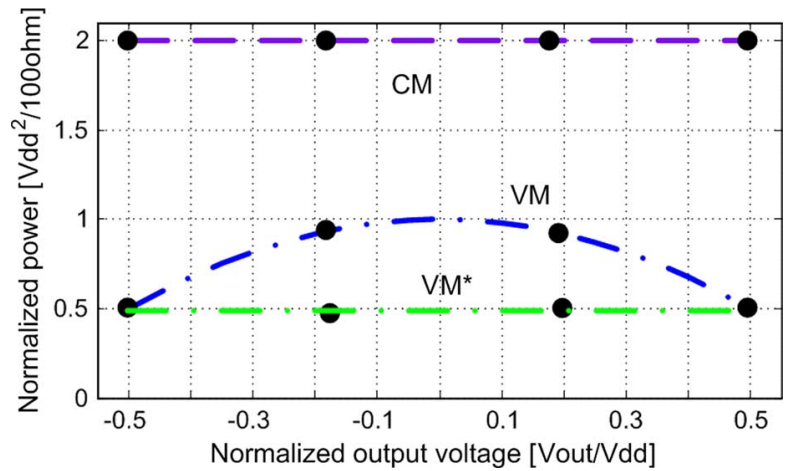

Fig. 4. Normalized static power characteristics for CM, VM, and VM* drivers.

drivers. The actual driver implementations sometimes follow this philosophy to avoid complex digital signal-processing, thus implementing each equalizer tap through dedicated driver segments [12], especially for short equalizers. As shown in (3d), (4h), and (5n), these dedicated tap-segments would be sized linearly for $\mathrm{CM}$ and nonlinearly for $\mathrm{VM}$ and $\mathrm{VM}^{*}$ drivers.

In Fig. 4, we compare the derived static power characteristics of $\mathrm{CM}, \mathrm{VM}$, and $\mathrm{VM}^{*}$ drivers normalized by $V_{\mathrm{unit}}=V_{\mathrm{DD}}$ on the voltage axis and $P_{0}=V_{\mathrm{DD}}^{2} / 100 \Omega$ on the power axis. The dots superimposed on the power characteristics of each driver are an example of a set of encoded output voltages produced when equalizing a particular channel, given by (1). Assuming uniformly distributed input data sequence, we seek to minimize the average power of these points, (2).

The $\mathrm{VM}^{*}$ driver can be further optimized if constant supply current is not necessary. In this case, a static power characteristic below that of $\mathrm{VM}^{*}$, Fig. 4, could be achieved by appropriate choice of transmitter resistances $\left(R_{a}, R_{b}\right.$ and $\left.R_{c}\right)$ for each encoded voltage.

The most interesting aspect of the $\mathrm{VM}^{*}$ topology is that it changes the approach to channel termination compared to $\mathrm{CM}$ and VM equalizers. While the idea behind $\mathrm{CM}$ and VM channel interfacing is to try emulating the nominal channel termination impedance, the $\mathrm{VM}^{*}$ driver is designed with a more realistic proxy for limiting pre-equalization ISI: a prescribed bound on CM reflected energy inside the Nyquist band of frequencies [6]. To see this, we note that, at low frequencies, we have (we assume that operator " "|" for parallel impedance has precedence over algebraic operations)

$$
\begin{aligned}
Z_{d m-L F} & =\left(2 R_{b} \| R_{a}\right) \| R_{c} \\
Z_{c m-L F} & =\left(R_{b} \| R_{a}\right)
\end{aligned}
$$

where $Z_{d m-L F}$ is the differential mode termination at low frequencies and $Z_{c m-L F}$ is the CM termination at low frequencies. At sufficiently high frequencies, we can assume that the impedance of $C_{a c}$ is sufficiently lower than the equivalent resistance it connects to, so we have a high-frequency version of (6a) and (6b) as

$$
\begin{aligned}
Z_{d m-H F} & =\left(2 R_{b} \| R_{a}\right) \| R_{c} \\
Z_{c m-H F} & =\left(R_{b} \| R_{a}\right) \|\left(R_{c} / 2\right) .
\end{aligned}
$$

It is obvious that not all of these termination impedances can be equal to the channel characteristic impedance at the same time (or any fixed impedance in general). The resistances $R_{a}$, 


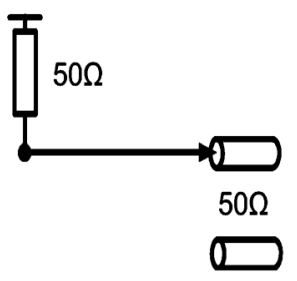

(a)

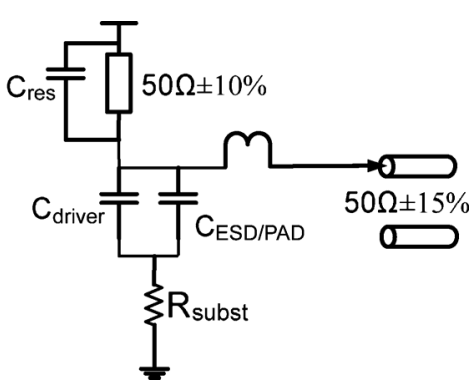

(b)
Fig. 5. (a) Nominal model. (b) Equivalent schematic of a termination network.

$R_{b}$, and $R_{c}$ are determined from the output voltage constraint analogous to the one in (4f) and by making the tradeoff between differential and CM impedance matching over the frequencies of interest. In particular, improvements in power efficiency, past the VM driver performance, can only be achieved if a more relaxed approach to impedance matching is adopted.

In the next sections, we make the next step in improving the power efficiency by further relaxing the impedance matching requirements (of both differential and $\mathrm{CM}$ ), but analyzing the performance impact with truly relevant metrics, such as equalized eye opening instead of loosely related indirect proxies.

\section{IMPEDANCE MATCHING IN LINKS}

Impedance matching and $S$-parameter masks are utilized in RF design for power transfer maximization from the transmitter to the line (conjugate matching) and for power absorption maximization by the antenna (reflectionless matching). None of these constraints directly relates to link design environment, yet they are present in all link standards.

Link receivers are not implemented through matched filters, since they operate in the high-SNR regime, and power transfer maximization done in RF systems is not necessary. Moreover, the power match is a narrowband concept well suited for cases where the signaling bandwidth is small compared with the carrier frequency. Finally, implementing a low impedance-matching network in deeply scaled CMOS technologies is challenging. Due to the transistor, wiring, and ESD parasitic capacitance and package inductance [Fig. 5(b)], the nominal impedance match [Fig. 5(a)] cannot be achieved in wideband systems such as high-speed links. Furthermore, the production tolerance of the channel and transistor characteristics puts even the nominal (low frequency) matching in question.

For example, the parasitic capacitance at the output of the transmitter is $\sim 400 \mathrm{fF}$ in recent, well-optimized designs [4], [5]. At a 7-Gb/s data rate, this parasitic capacitance leads to termination impedance $1 /(50 \Omega \|(\mathrm{j} 127 \Omega))$ at Nyquist frequency, which is significantly different than the nominal $50-\Omega$ termination, even without production tolerance effects.

From these considerations, we see that impedance matching (and more generally $S$-parameter masks) is intuitive but qualitative proxy for describing signal quality, not a fundamental constraint for proper operation. It is an idealization, geared towards

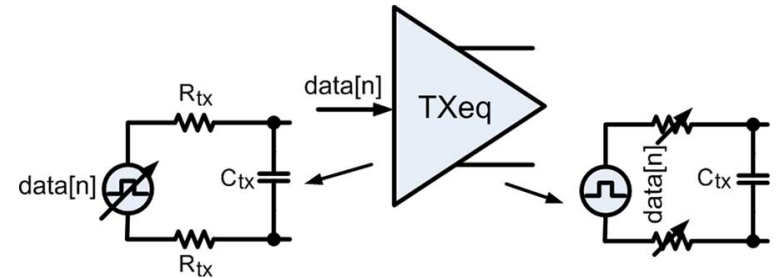

Fig. 6. System model of a transmit equalizer for an impedance matching and impedance modulating case.

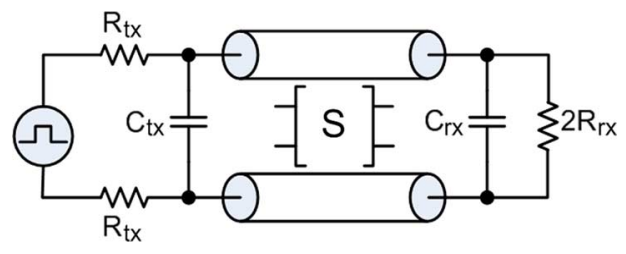

Fig. 7. Equivalent schematic for studying impedance matching and modulation in links.

ease of standardization and manufacturing, and in view of practical obstacles for achieving this perfect matching we proceed to explore the possibility of energy savings if this constraint is removed. We do this by evaluating the dependence of the ultimate link performance metrics such as energy efficiency (pJ/bit) and a given bit error rate (BER) — or equivalent eye opening.

\section{IMPEDANCE MODULATION}

At the system level, the transmit equalizer has a very simple model shown in Fig. 6. If the impedance matching constraint is to be observed the equalization must be implemented by a controllable voltage source, resulting in the discussed driver topologies.

On the other hand, if we abandon the impedance-matching constraint, the encoded voltages needed for equalization can be generated by making the transmitter resistance controllable, shown in Fig. 6, and programming the resistive divider between the transmitter termination and the channel/receiver (Fig. 7). We refer to this driver as the resistance-mode (RM) driver [7].

To understand RM drivers, we must first study the behavior of the channel under dynamically changing transmitter resistance. However, there is no simple solution to this problem since the channel becomes time-variant and data-dependent. Since the system changes parameters dynamically and depends on data, the time-invariant linear analysis is not possible as transfer function cannot be defined. Because of this, we resort to simulation and experiment to build intuition about the behavior of these systems.

In the ideal case, the transfer function between the voltage source and the channel's input in Fig. 7 would be a simple, realvalued (frequency-independent) attenuation. The energy flow would occur only from the transmitter to the receiver and there would be no difference between matched and unmatched transmitter except in attenuation level. However, with imperfections in the channel (e.g., package or connector via discontinuities) and imperfect matching due to parasitic elements (e.g., ESD capacitance, etc.) reflections will exist in the channel and they will 


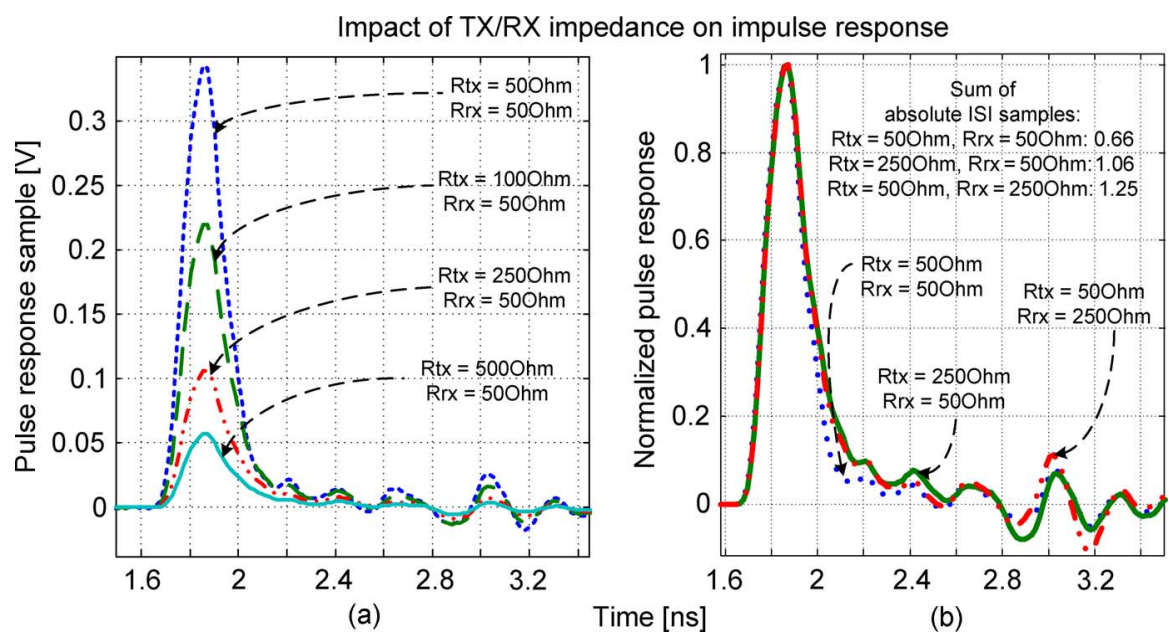

Fig. 8. (a) Pulse response of B3-a 3-in channel versus $R_{\mathrm{tx}} / R_{\mathrm{rx}}$ interface resistance at $6.25 \mathrm{~Gb} / \mathrm{s}$. (b) Normalized pulse response for $\mathrm{B} 3$ channel to eliminate the scaling versus $R_{\mathrm{tx}} / R_{\mathrm{rx}}$; the loss at Nyquist frequency is $15 \mathrm{~dB}$.

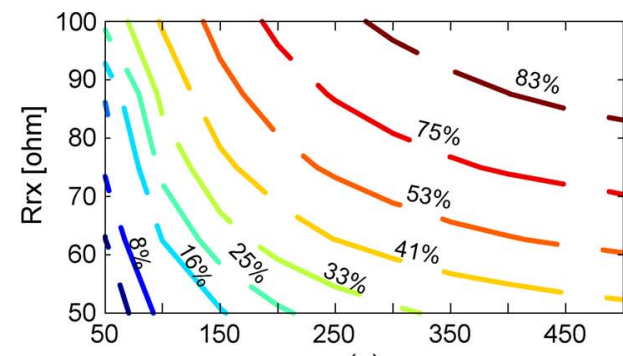

(a)

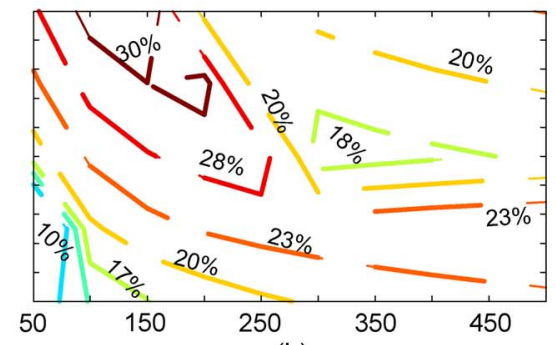

(b)

Fig. 9. Relative degradation of the worst-case eye equalized by 3-tap transmit pre-emphasis filter as a function of transmit and receiver interface resistance for (a) B3 3-in backplane channel and (b) B32 32-in backplane channel [9].

cause signal degradation and increase in ISI if the transmitter is not impedance matched and does not absorb them.

We first explore the impulse response dependence on transmit and receive termination impedance, assuming that they are fixed. The first step is obtaining the transfer function, which is easiest to calculate from $Z$ - or $Y$-parameters. To do this, we use the measured $S$-parameters of a channel and convert them to $Z$-parameters [13] as

$$
Z(\omega)=Z_{0} \frac{I+S(\omega)}{I-S(\omega)}
$$

where $Z_{0}$ is the termination matrix during measurement, $\omega$ is frequency, and $\mathbf{I}$ is the identity matrix. In this form, it is easy to add impedances in series or in parallel with the channel on either end, and by sampling in frequency it is possible to obtain the impulse response through inverse discrete Fourier transform (IDFT).

For simulations we first use B3, a 3-in backplane channel [9], as a conservative estimate, because it has low loss and shows more degradation due to impedance mismatch and reflections. In Fig. 8(a), we can see dependence of a $6.25 \mathrm{~Gb} / \mathrm{s}$ pulse response on $R_{\mathrm{tx}}$ and $R_{\mathrm{rx}}$, assuming $C_{\mathrm{tx}}=C_{\mathrm{rx}}=300 \mathrm{fF}$ (or 600-fF single-ended parasitic capacitance). To provide a fair comparison of the signal quality, Fig. 8(b) shows normalization of the responses, where the scaling due to voltage divider was removed. In Fig. 8(b), we also calculated the absolute sum of all the ISI samples for these three termination scenarios. It can be observed that the dominant influence on reflections is from the receiver termination [Fig. 8(b)], while the transmitter side increases dispersion and scales the response due to the resistive divider shown in Fig. 7.

To visualize the signal degradation from additional reflections and dispersion in Fig. 9(a) and (b), we show relative degradation of the worst-case eye-opening as a function of transmit and receive termination resistance. In Fig. 9(a), we can see the B3 channel, while Fig. 9(b) shows results for more lossy, B32-a 32-in backplane channel [9]. To remove the scaling, introduced by the transmitter termination, we use the normalized pulse responses. The lossy B32 channel shows significantly less degradation as the possible reflections from the receiver experience twice the attenuation of the channel until they appear at the output. In this scenario, the receiver impedance can be increased to a certain extent to increase the gain of the system. Finally, we note that, in practice, when using dynamic impedance modulation, we can expect less degradation than what is indicated in Fig. 9. This is because in Fig. 9 we see an estimate of the worst-case scenario since we assume always having the same impedance connected and modulating the equivalent voltage source of the equalizer.

On the other hand, when using RM driver, the impedance at the transmitter end changes as a function of the incoming bit-stream. Typically, when equalizing, the high impedance will be presented to the channel only when long streams of zeroes/ ones arrives to the transmitter thus prompting the equalizer to 


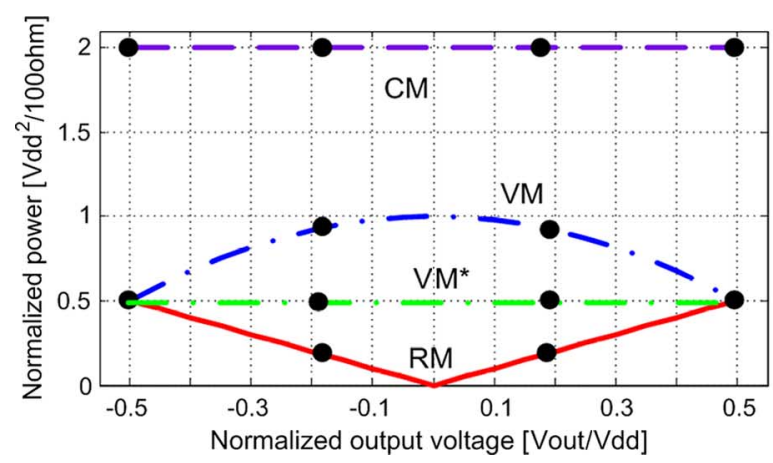

(a)

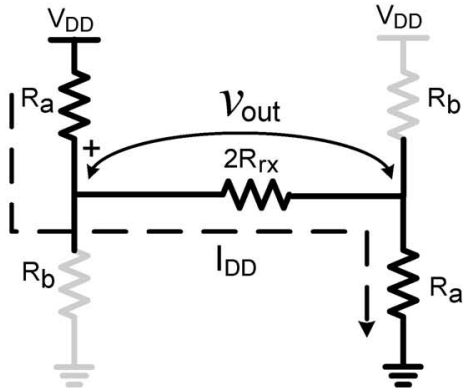

(b)

Fig. 10. (a) Comparison of the RM driver static power characteristics with CM, VM, and VM*

. (b) Equivalent schematic of the RM driver.

use low output voltage. This is the configuration that can, potentially, produce the strongest reflection and the largest degradation of the signal. However, for this to happen, a reflection from a connector or receiver needs to arrive approximately at the same time to the transmitter. This happens very rarely, only when the bit-sequence was very active one reflection round-trip time ago. So, for a range of output impedances used in RM-modulation, we can expect levels of signal degradation bounded by the values in Fig. 9, but certainly less than the worst case defined by the highest impedance in the range. As the channel is low-pass, we can expect that time-varying transmit impedance would be 'interpolating' between the impulse responses shown in Fig. 8. In this mode, time-invariant transfer function of the system does not exist since poles of the system change due to changes in the transmitter impedance. However, by designing the range of these impedances such that the lowest position of the moving poles is always well beyond the $3-\mathrm{dB}$ bandwidth of the channel's dominant pole, the moving poles can be neglected, thus enabling us to control the signal amplitude and achieve equalization.

The power characteristic of the RM driver can be simply calculated by observing, in Figs. 7 and 10(b), that this topology always steers all of the current from the transmit supplies through the receiver without any shunting. As we assume maximum output voltage of $V_{\mathrm{DD}} / 2$, at its maximum power this driver must have the same power consumption as the VM driver. Thus, we can write

$$
P_{\text {out }}\left(V_{\text {out }}\right)=\left(\frac{V_{\mathrm{DD}}^{2}}{100 \Omega}\right)\left|\frac{V_{\text {out }}}{V_{\mathrm{DD}}}\right| .
$$

The pulse scaling, observable in Fig. 8(a), as a result of transmit-side impedance change can be expected to follow the compression curve for the resistive divider between the transmitter and receiver resistance, as seen in Fig. 10(b):

$$
v_{\text {out }}\left(R_{\mathrm{tx}}\right)=\frac{R_{\mathrm{rx}}}{R_{\mathrm{rx}}+R_{\mathrm{tx}}} V_{\mathrm{DD}} .
$$

The RM driver power characteristic is shown in Fig. 10(a) along with the power characteristic of $\mathrm{CM}, \mathrm{VM}$, and $\mathrm{VM}^{*}$ drivers, for comparison. It can be seen that RM driver creates bit-to-bit current variations of the same amplitude as the VM driver, and in that regard the two are comparable. The only solution to this problem is appropriate supply decoupling, preferably inside the driver layout.
It is interesting to note that, even though the $\mathrm{VM}^{*}$ driver can achieve a power characteristic below the curve given by (5a) and shown in Fig. 10, as we discussed earlier it cannot be strictly superior to the RM curve, anywhere, as it will always have some current leakage through the differential switch, as shown in Fig. 3. Furthermore, $\mathrm{VM}^{*}$ implementation would introduce almost twice as much parasitic capacitance at the equalizer output. It would, also, have more complicated predriver and decoder due to all the additional segments. Similarly, a redesign of CM driver without transmitter-side termination, sometimes referred to as open-drain $\mathrm{CM}$ driver, can be used to improve the performance of CM-type drivers. Nominally, such driver would have approximately $2 \times$ better power efficiency when compared with the standard CM-type driver we described. However, due to missing transmitter-side termination the driver suffers from impulse response degradation due to reflections, larger than the RM driver, and thus exhibits less than $2 \times$ improvement in power efficiency (at same performance level) when compared to the standard $\mathrm{CM}$ driver.

Finally, we note that RM driver pull-up/pull-down control signals for each (differential) side are simple inversions of each other. On the contrary, $\mathrm{VM} / \mathrm{VM}^{*}$ drivers always have some portion of the pull-up/pull-down and differential switches turned on thus requiring separate decoding for each of them, leading to additional decoder complexity, regardless of the actual implementation strategy (e.g., if we use a lookup table (LUT) or some other decoder form). This difference becomes especially pronounced as the number of taps (filter order) is increased.

\section{Proposed System ArChitecture}

The tap-segmentation approach, while popular for very short equalizers, increases the total driver size and hence parasitics at the output node (and therefore introduces both power penalty and performance degradation), since each tap has to be sized for the worst-case range over the whole spectrum of channels in the intended application. While some advanced tap-sharing methods have been developed [12], the most efficient approach to reducing the output parasitics and keeping the equalizer flexibility is to implement a linearly segmented driver, basically a general purpose digital-to-analog converter (DAC). This comes at a cost of digital encoding circuits, which can be mitigated with appropriate back-end design style. 


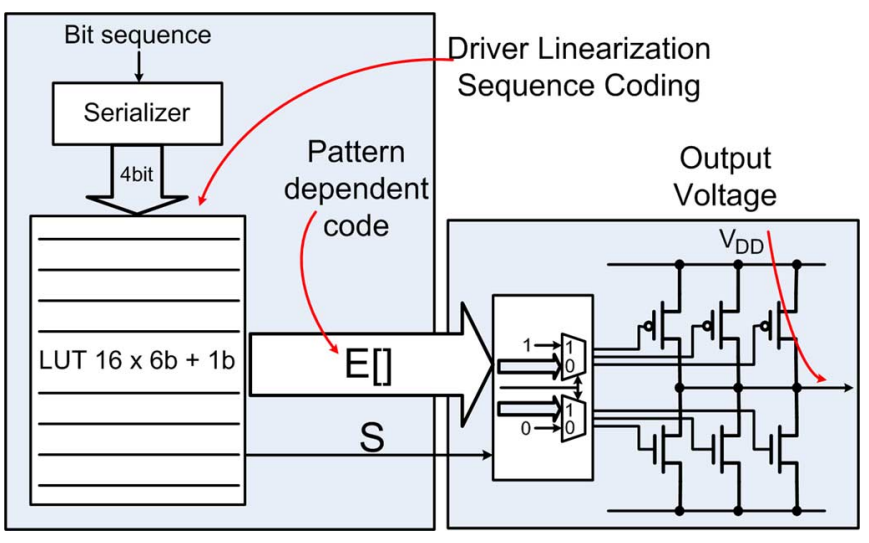

Fig. 11. Proposed RM equalizer system architecture.

While the digital encoding necessary to achieve tap programmability can be implemented in various ways, in this implementation, we choose a LUT approach since it offers the most flexibility in terms of driver linearization and equalization. As discussed in previous sections, in addition to computing the segment encoding that corresponds to the strength of the equalized sample, the encoding block should ideally also compensate the nonlinearity of the output driver, as seen in the analysis of $\mathrm{VM} / \mathrm{VM}^{*}$ and $\mathrm{RM}$ driver, (4f), (5n), and (9).

The overall system architecture for the four-tap (third-order) RM equalizer is shown in Fig. 11. The range over which the RM impedance is modulated represents a tradeoff between signal quality and energy efficiency. Since our ultimate metric is the eye opening at the receive side, for any given channel, there exists a range of RM impedances that will yield the lowest energy for same receiver eye opening constraint. To experiment with these ranges and allow flexible equalization, we implement the RM equalizer as a simple, segmented CMOS tri-state buffer.

Since the segmented driver is fully digitally controlled, a programmable dynamic pattern LUT is implemented in the backend, to facilitate the channel equalization, similar to [10]. The LUT stores digital representations of the bit-pattern-dependent output voltages. We will show shortly that the LUT back-end can also be used to compensate the driver nonlinearities [like the systematic compression in (9)] and other undesirable effects such as duty-cycle mismatch. This also decouples the driver design complexity from the number of taps and minimizes the output parasitic capacitance by reducing the number of driver transistors as it eliminates the overhead of the tap-segmented architectures.

The driver is designed as a segmented push-pull pseudo-differential CMOS bridge, as shown in Fig. 11. For improved segment matching, all of the segments have the same size. They also share the same sign signal (S), but have separate enable (E) signals, thus giving the full control of the sign and absolute value of the output voltage. The enable vector $\mathbf{E}$ (Fig. 11) controls the transmitter resistance $R_{\mathrm{tx}}$ between the supply and the load (Fig. 7).

In order to reduce the LUT size, the filtered and predistorted voltages are stored as $1 b+6 b$ sign and absolute value and appropriately decoded to produce the sign (S) and enable signals (E[]) needed by the driver.

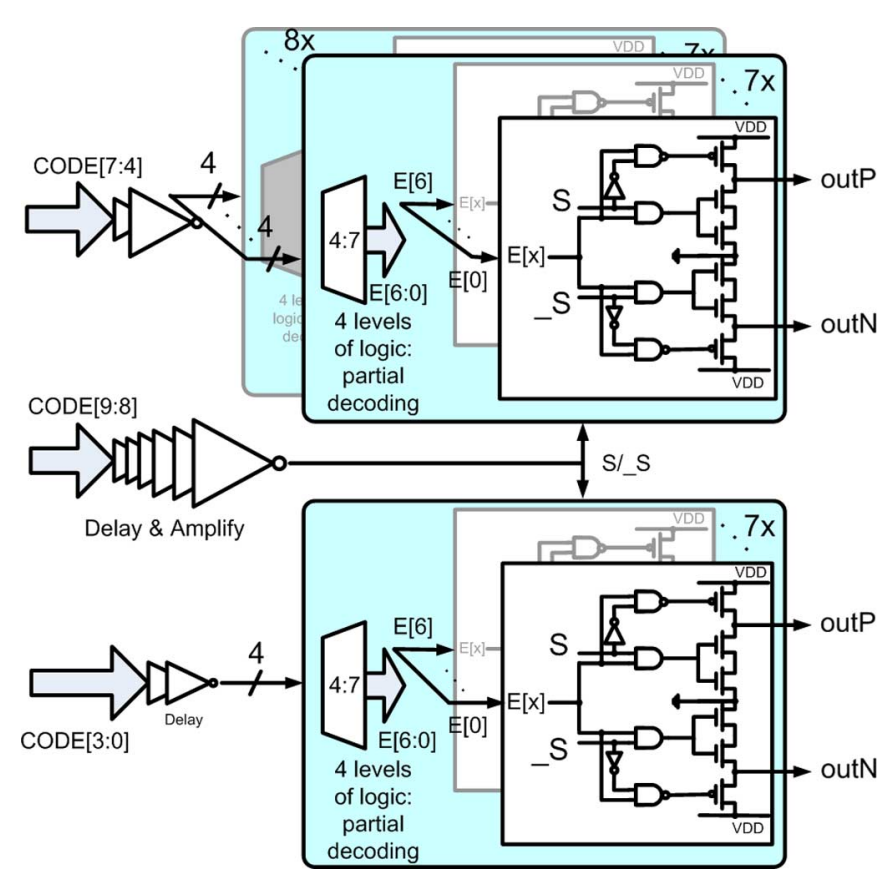

Fig. 12. Implementation of the RM output driver.

In our particular implementation, we choose to implement 63 driver segments for 6-bit output value encoding. The total driver output impedance ranges from $50 \Omega-3.15 \mathrm{k} \Omega$. A full thermometer coded driver would be preferable from a power-efficiency perspective, as it minimizes the switching activity of all of the RM driver segments. However, implementing a full thermometer decoding would increase complexity and size of the driver layout, as well as bring significant routing problems and wire capacitance as all of the decoded segment controls would need to be routed to the driver output. An alternative approach, enabling a compact and regular layout, was chosen by segmenting the driver into eight tiles of seven slices each, controlled by the higher $3 \mathrm{~b}$ of the LUT code, and one seven-slice tile controlled by the lower $3 \mathrm{~b}$ of the LUT code. In other words, a segmented thermometer coding of the output slices is implemented. The actual number of active slices, given a $\operatorname{code} c[5: 0]$, is

$$
n(c[5: 0])=2^{3} c[5: 3]+c[2: 0]
$$

resulting in the termination resistance dependence on the LUT code given in

$$
R_{\mathrm{tx}}(c[5: 0])=\frac{3.15 \mathrm{k} \Omega}{n(c[5: 0])} .
$$

The implementation is shown in Fig. 12.

The LUT addresses are formed from the incoming bit sequence in the serializer and decoded into the multiplexer control signals as shown in Fig. 13(a). To enable fast readout of the LUT tables, a dual-data-rate (DDR) topology of the digital backend was chosen. It is important to note that LUT entries are precalculated values and can be stored in a static structure without the need for real memory. Each LUT is a 16:1 7-bit-wide multiplexer designed as two cascaded 4:1 multiplexer stages in passtransistor logic, as seen in Fig. 13(b) for one of the DDR phases. 


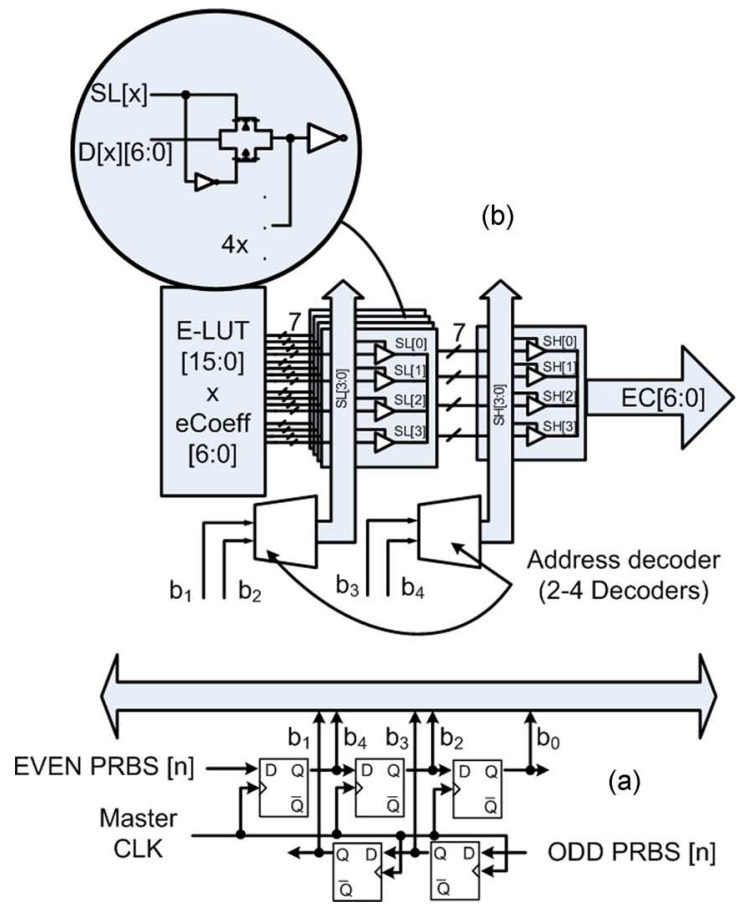

Fig. 13. Implementation of digital backend for the RM equalizer.

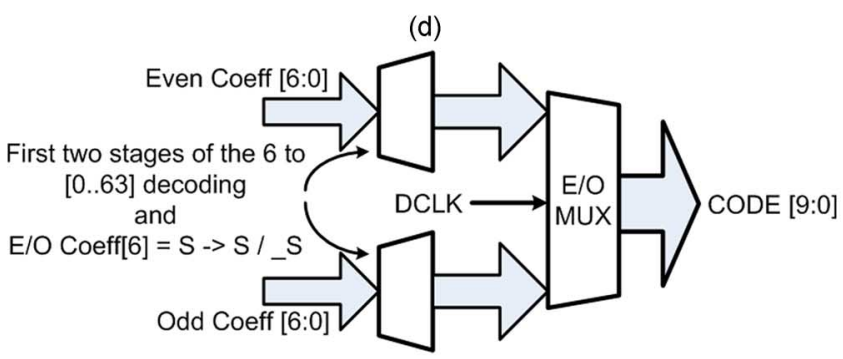

(c)

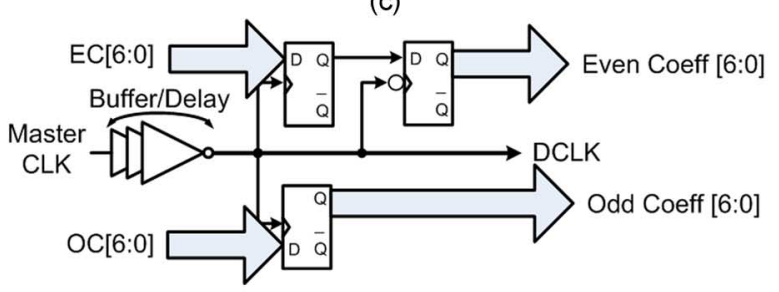

The master clock is delayed through a chain of inverters for use with even/odd (E/O) latches, shown in Fig. 13(c). The delay of the clock is meant to compensate for the delay of the address decoding and memory readout, thus relaxing the setup time constraint on the E/O latches.

The decoding from the binary representation $c[5: 0]$ to $\mathrm{E}[62: 0]$, would be usually done either before or after the $\mathrm{E} / \mathrm{O}$ multiplexer. However, in our design the $\mathrm{E} / \mathrm{O}$ multiplexer is inserted inside the decoder block, shown in Fig. 13(d). This approach relaxes the latency constraints on the decoder, as only the first few stages of the decoder need to evaluate by the next clock edge. On the other hand smaller number of decoder stages after the E/O multiplexer reduces the jitter accumulation in these unclocked stages of logic.

Splitting the decoder into two parts also made it easier to match the propagation delays through various decoder logic chains and ensure that slices change state within a narrow interval of time. This produces a monotonic, well-defined symbol edge. Since sign signal $\mathrm{S}$ and its inverse are shared among all of the tiles, the signal is delayed and amplified appropriately to arrive approximately at the same time with the enable signals (E) to all the segments (Fig. 12).

The proposed structure is completely digital and can be synthesized. The only part of the circuit that needs special attention is the output driver slice, where the two sides (pull-up pMOS and pull-down nMOS transistor) need to be matched to provide a well-balanced common mode. In a sense, the implemented structure is very similar to the transmitter reported in [11], but derived from different considerations. While the system described in [11] is implemented for on-chip applications, it could be analyzed as a particular implementation (with predetermined equalization) of the RM-type driver. The link in [11] achieves significantly better energy efficiency in an on-chip $R C$-limited global interconnect application which is vastly different than the chip-to-chip applications where the channels are designed in $R L C$ regime and are much more sensitive to impedance discontinuities. In on-chip $R C$-limited wire scenarios, the transmitter has significantly less capacitive loading as the pad capacitance is not present for the on-chip application in [11], and the output transistors of the driver are sized for much higher impedances since, due to the high resistive losses, the channel in that application does not have reflections of any kind. Since the on-chip channel varies very little in range of transfer functions, the equalizer in [11] is designed as a three-tap filter with direct state-machine encoding and narrow-range static nonlinear segmentation. In off-chip applications, the equalizer has to be more flexible, due to varying channel characteristics, hence in this work we explored the challenges in implementing a longer four-tap equalizer and LUT-based encoding back-end.

\section{Measurement Results}

A prototype transmitter to test the ideas described in previous sections was fabricated in a 90-nm CMOS process. In Fig. 14, we show the die photograph and the layout of the test chip. The transmitter area is $150 \mu \mathrm{m} \times 130 \mu \mathrm{m}$. The decoupling capacitors, implemented as gate capacitors, can be seen next to the output driver. In our experiments, we did not encounter any problems with the driver supply, with the indicated local supply decoupling of approximately $20 \mathrm{pF}$. This value should be compared with the CM termination capacitance $C_{a c}$ that, together with the differential termination switch resistance $R_{c}$, sets the low-frequency $\mathrm{CM}$ termination of the $\mathrm{VM}^{*}$ driver [6]. To provide a good ac ground at $50 \mathrm{MHz}$, due to the $S$-parameter mask specification at $50 \mathrm{MHz}$ and above [6], this capacitor would have to be at least the same size as our decoupling capacitance, assuming that $R_{c}$ is of the order of $100 \Omega$. 


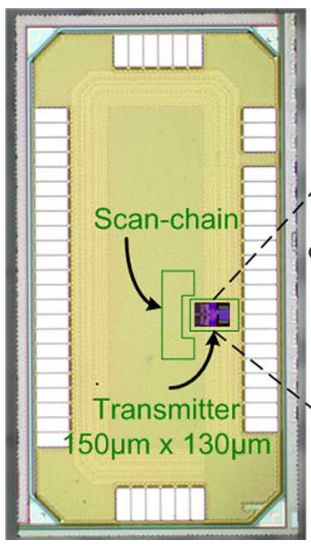

(a)

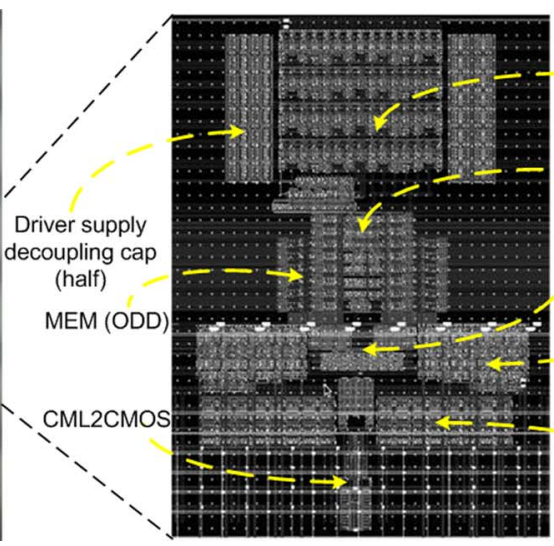

RM driver

E/O MUX and partial

Decoding

$\mathrm{EC}[6: 0]->\operatorname{CODE}[9: 0]$

Serializer

31-bit PRBS (EVEN)

PRBS supply decoupling cap (half)

(b)

Fig. 14. (a) Testchip die photograph. (b) Layout of RM transmitter.

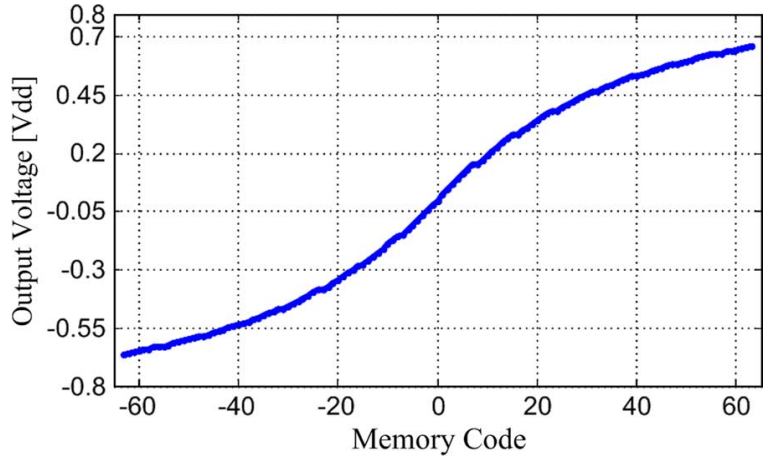

Fig. 15. Compression curve of the RM DAC driver.

In our experimental setup, we assume capacitive (ac) coupling of the channel. Equivalently receiver side channel interface should have high impedance to both ground and supply, and $100-\Omega$ differential termination with no more than $\sim 300 \mathrm{fF}$ of equivalent differential capacitance. Two 31-bit PRBS generators (one for each DDR channel) with independent seeds were implemented for data generation. All the measurements were performed with $V_{\mathrm{dd}}=1.15 \mathrm{~V}$.

The first measurement performed is the compression curve of the output stage relating the LUT codes with the desired output voltages. This calibration step could even be integrated with the transmitter itself by sweeping memory codes and measuring the produced voltages at the transmitter output during the initialization phase of the transmitter. Since the conductance of the transmitter is proportional to the memory code, this measurement shown in Fig. 15 is a simple confirmation of the shape of the compression curve given in (9). Once the equalization encoded voltages for a specific channel are known, this compression curve is used to determine the appropriate LUT entries.

In Fig. 16, we show the eye diagrams of the equalized and unequalized ac-coupled link running at $4 \mathrm{~Gb} / \mathrm{s}$ over 26 in of the FR4 backplane. At this speed, the eye is equalized with effectively a two-tap pre-emphasis filter, pre-distorted to correct for the DAC nonlinearity and mapped into the LUTs.

Fully independent LUTs in the digital backend ( Fig. 12) allow for static timing offset correction in the transmitter. To

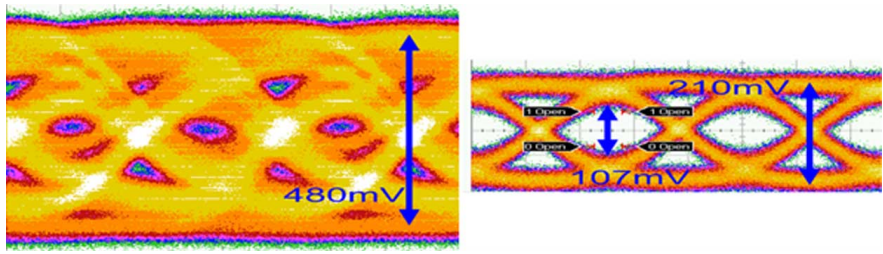

Fig. 16. Equalization of the 26-in FR4 channel at $4 \mathrm{~Gb} / \mathrm{s}$.
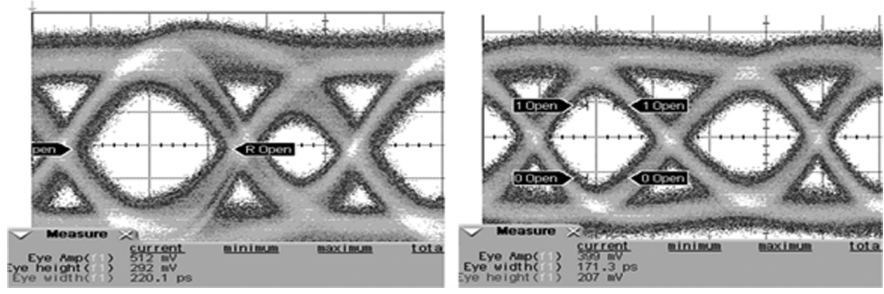

Fig. 17. Receive eye diagrams showing joint pre-emphasis and duty-cycle correction with the RM driver LUT.

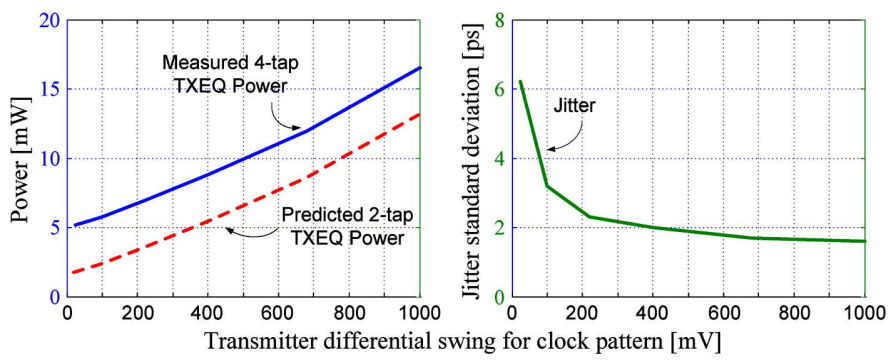

Fig. 18. Power and jitter versus output swing for the clock output pattern.

illustrate the effect of duty-cycle compensation, we distort the duty cycle of the external link clock so that even/odd eyes at the receiver are $292 \mathrm{mV} / 226 \mathrm{ps}$ and $128 \mathrm{mV} / 113 \mathrm{ps}$, respectively. By scaling down the symbols in one of the LUTs, the eye diagrams are balanced with $207 \mathrm{mV} / 171 \mathrm{ps}$ and $198 \mathrm{mV} / 178 \mathrm{ps}$, in Fig. 17.

The transmitter power is illustrated in Fig. 18, showing the decoder path overhead of $5 \mathrm{~mW}$ at $4 \mathrm{~Gb} / \mathrm{s}$, and linear scaling of driver power with output swing, as predicted in Fig. 10. Due to 


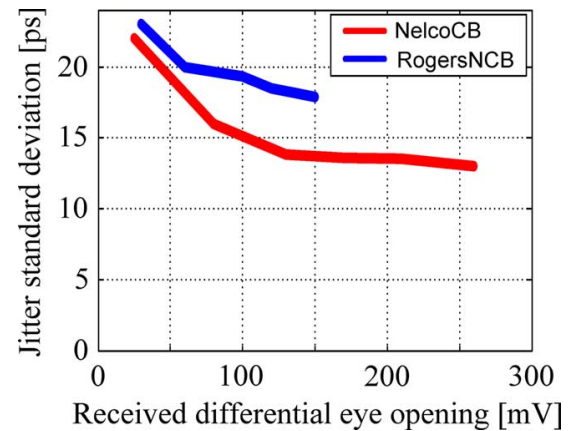

Fig. 19. Zero-crossing jitter of the equalized eye diagram; channels are 20-inlong backplane channels.

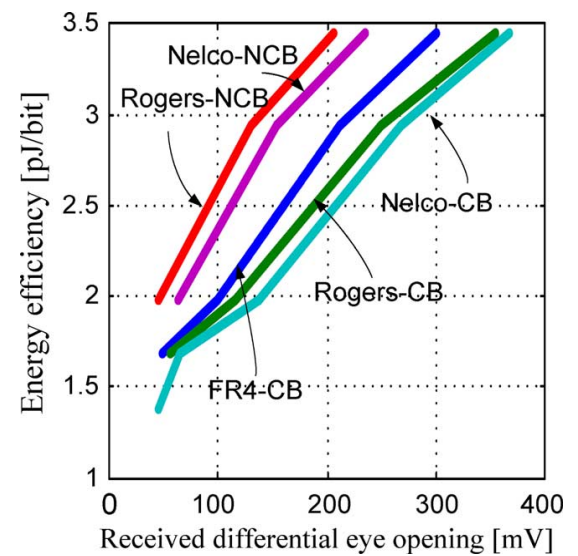

Fig. 20. Energy-efficiency of the RM driver over various backplane channels with Nyquist frequency loss of $8 \mathrm{~dB}$ to $12 \mathrm{~dB}$.

a design error in transition from the serializer to the LUT address decoder, the LUT range was reduced to 2-bit instead of 4-bit address. Transmitter speed was also reduced due to relatively large load the address decoder presents to the serializer's quasi-minimal output buffers. As the second-order LUT would have been $\sim 4 \mathrm{x}$ smaller, we can expect that the decoder overhead (Fig. 18) would have been $1.5-2 \mathrm{~mW}$ in this case, bringing us to $\sim 1.25 \mathrm{pJ} / \mathrm{b}$, for the $100-\mathrm{mV}$ eye opening.

In Fig. 19, we show the edge jitter in the equalized eye diagram versus the eye opening for the best and the worst channel we had available for measurement (the noncounterbored (NCB) and counterbored (CB) low-loss channels). As the received differential eye is scaled down, the residual ISI is increased and the zero crossing jitter increases as well. There are two main reasons for this. First, signal scaling is achieved by increasing the transmitter impedance, leading to more reflections from the transmitter and added ISI. Second, due to the compression characteristic in Fig. 15, as we scale down the encoded output voltages, the roundoff errors in coefficient quantization increase, leading to degradation in equalization and increase in ISI. The jitter degradation seems to follow the transmit jitter curve in Fig. 18.

Fig. 20 shows the energy efficiency of the equalization over backplane channels with 8-12 $\mathrm{dB}$ loss at Nyquist frequency of $2 \mathrm{GHz}$, from low-loss Nelco and Rogers channels, with and without counterboring to lossy FR4 channel. As expected, the equalizer performance is the best on dispersive channels and

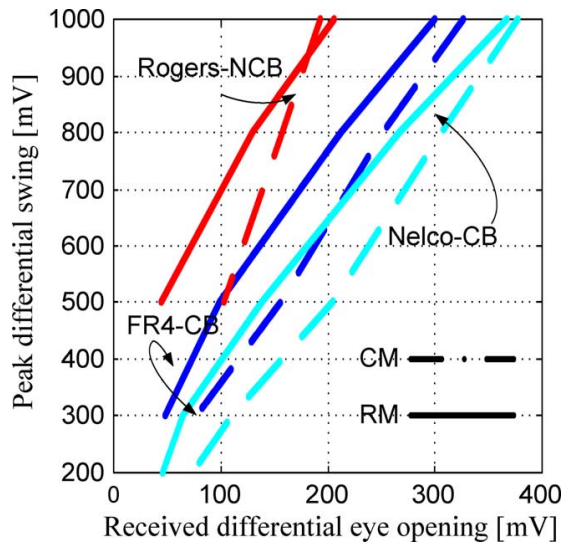

Fig. 21. Comparison of transmitter swing requirements for RM and $\mathrm{CM}$ drivers given the measured receiver eye opening.

the worst on low-loss channels without counterboring, where transmit pre-emphasis cannot equalize distant reflections from long backplane via stubs. The CM fluctuations were spread out evenly through the symbol period in these measurements and scaled linearly from 30 to $75 \mathrm{mV}$ for eye openings in Fig. 20 .

The power-efficiency degradation observed when the RM driver operates over low-loss reflective channels comes from the additional faraway reflections that arise due to the impedance mismatch of RM driver and the channel. The reflections are actually initiated by the channel impedance discontinuities (e.g., connectors) but reflected by the transmitter due to its impedance mismatch. Since noncounterbored channels have bigger impedance discontinuities, these channels produce higher reflections-based residual ISI. As these reflections happen to be outside of the equalization scope, the only way to neutralize their effect is to scale up the encoded voltages used in the RM driver. Measurements of the received eye opening for a given peak differential transmitter swing are given in Fig. 21. They can be used to calculate the exact scaling necessary to apply in RM driver to achieve the same performance as a fully impedance-matched CM driver.

\section{Signal Quality Versus Power-EFficiency TRAdeofF}

As expected, Fig. 21 shows that RM driver will always have to operate at larger output voltages, when compared with the matched drivers, in order to compensate for the eye degradation due to the additional reflections. This means that our intuitive picture of the equalization cost in RM driver as compared to other, matched, drivers, shown in Fig. 10, needs to be refined.

In Fig. 22, we update our model to account for the necessary scaling of the coefficients used in RM driver when a fair metric, such as the same receive eye-opening is used for all the drivers. Note that encoded voltages, depicted by the black dots in the figure, have the same relative ratios in the RM driver case as in all other cases. The only parameter that changes in the RM case is the peak swing. The actual scaling factor for the peak swing of RM driver is a complicated function of the actual voltage levels transmitted, due to the dependence of reflection-induced degradation on the actual impedance of the driver. One convenient way to parameterize this dependence is through the achieved eye opening, assuming the optimal equalization for the given 


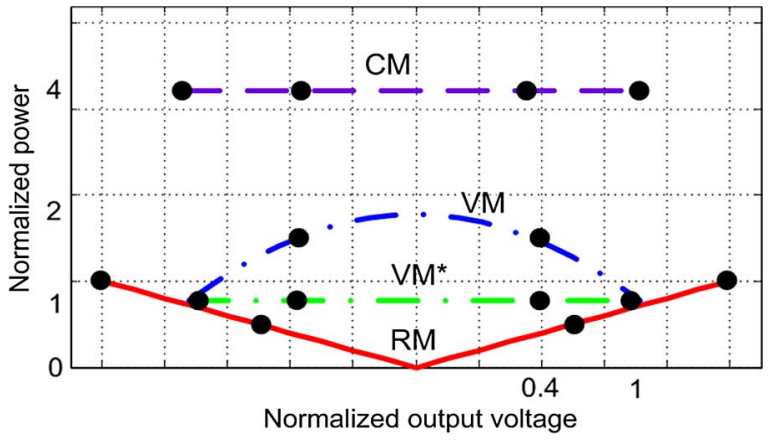

Fig. 22. Power characteristic comparison when all of the drivers are producing the same eye opening at the receiver.

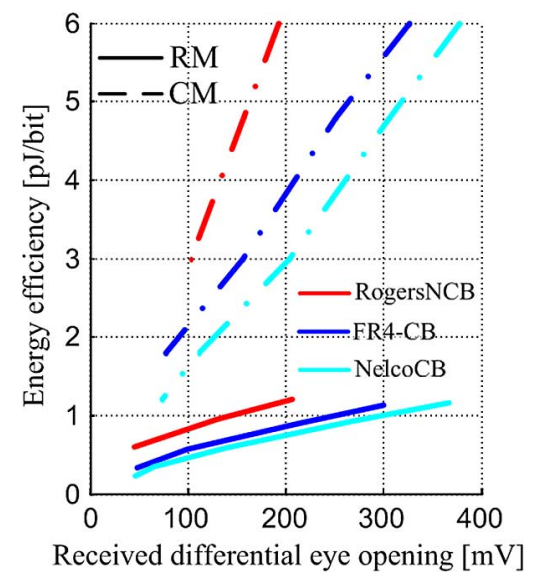

Fig. 23. Energy efficiency comparison of the $\mathrm{CM}$ and the RM driver, based on measured receiver eye opening for $\mathrm{V}_{\mathrm{DD}}=1.15 \mathrm{~V}$.

settings, as was done in Fig. 21. Thus, for every received eye opening, a diagram similar to Fig. 22 can be constructed, where the scaling between RM and other constellations, for a certain received eye, is given in Fig. 21.

Since the encoded voltages scale accordingly, the average power is increased by the same factor. Using the measured swing overhead needed by the RM driver we can recalculate true link metrics, $\mathrm{pJ} / \mathrm{bit}$, for the RM and $\mathrm{CM}$ drivers. The results are shown in Fig. 23, where we estimate the output driver power (without the equalization backend) from the swing measurements in Fig. 21 and (3d) and (8) and Fig. 22. We can see that the RM driver now outperforms CM driver by approximately $5.5 \times$ for large receiver eye openings and about $3.5 \times$ for lower eye openings. This is because, at lower eye openings, the RM driver operates in higher impedance mode constantly, thus increasing the reflections and suffering the signal degradation more than in the case when larger swings are needed and the impedance range used is closer to the matched case.

To provide a reasonably fair comparison with $\mathrm{VM} / \mathrm{VM}^{*}$-type drivers, we are assuming that they would perform as well as the $\mathrm{CM}$ driver when measured against the RM driver. In particular, we assume the same ratio of necessary swings for the given receiver eye, Fig. 21. This is optimistic since $\mathrm{VM} / \mathrm{VM}^{*}$ have significantly more parasitic capacitance at their output than does the CM driver. We also do not penalize $\mathrm{VM}^{*}$ driver for the signal degradation arising from the common-mode-to-differential mapping in the channel

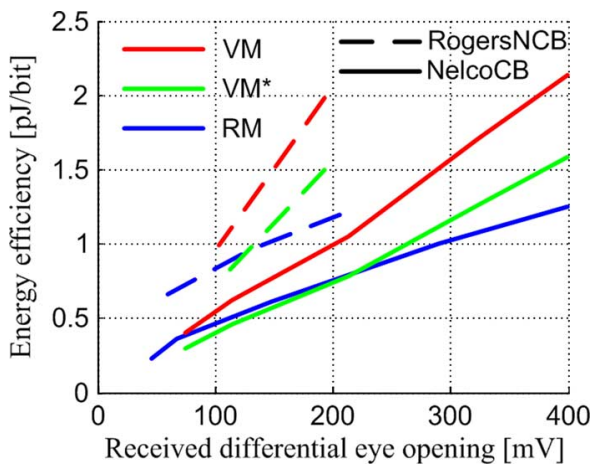

Fig. 24. Energy-efficiency comparison between the RM driver and the VM/VM* ${ }^{*}$, assuming appropriately scaled and regulated supply for each driver.

that arises from imperfect $\mathrm{CM}$ matching in $\mathrm{VM}^{*}$ drivers. This effect is fully included in RM driver performance prediction as the voltage overhead was directly measured. Furthermore, as most of the VM-type drivers operate from a lower supply voltage, we are going to assume a linear regulator for driver supply adjustment in all cases, including RM driver. In this case, the regulator provides the average driver current and the current spikes are handled by the decoupling capacitors.

The results of our extrapolation are shown in Fig. 24. We can see that, for high swings (receiver eye openings), the RM driver outperforms both $\mathrm{VM}$ and $\mathrm{VM}^{*}$ drivers. At lower swings, the RM driver's efficiency is about the same as that of the $\mathrm{VM}^{*}$ driver. Note that Fig. 24 compares only the driver performance, and it does not take into account complexity of decoder and pre-driver needed to implement equalization with a given driver. From this perspective, the RM topology is simpler to implement and requires less logic in the backend.

We should note that all of the explorations and predictions presented here were made by considering the measurements from the implemented RM driver sized for a certain supply voltage. Further improvements of the proposed topology can be made by considering lower supply voltage for the driver and appropriately sizing the driver transistors for operation from such a supply. This is often done for VM-type drivers where the output transistors are tuned for a certain supply voltage to achieve proper operation and tight control of the transistor impedance. In a sense, the implemented driver is an extreme in the space of all possible drivers once the impedance matching constraint is relaxed and included as a "soft" constraint through the power-versus-signal-degradation analysis.

\section{CONCLUSION}

Through analysis of various driver topologies and experimental results, this work shows that most energy-efficient transmit equalizers can be built by using drivers with dynamic impedance modulation. The range of this impedance modulation has to be chosen carefully to optimize the energy efficiency under true signal quality constraints (e.g., receiver eye opening), rather than impedance matching or return loss masks.

The removal of impedance matching constraints can lead to most energy-efficient designs for same performance constraints, 
since impulse response degradation due to the transmitter termination mismatch can be ameliorated through equalization and signal amplification. Furthermore dynamically modulating driver impedance greatly simplifies the transmitter design and saves significant amount of energy. Due to the predominantly low-pass nature of the link channel, this dynamic impedance modulation boils down to a linear system preceded by the memoryless compression curve.

Experimental results indicate that the transmit equalizer with RM driver and LUT back-end fabricated in 90-nm CMOS effectively equalizes various backplane channels at $4 \mathrm{~Gb} / \mathrm{s}$. It outperforms $\mathrm{CM}$ by at least $3.5 \times$ and VM by $2 \times$ in energy efficiency. At the same time, it achieves at least the same performance as the $\mathrm{VM}^{*}$-type driver while offering simpler and more compact implementation and significantly lower parasitic capacitance at the output.

The presented analysis method in the normalized driver power versus normalized output voltage space can be used to analyze and design all types of drivers, including new ones having performance between the $\mathrm{VM}^{*}$ and $\mathrm{RM}$ driver, that would be of possible interest in future applications.

\section{REFERENCES}

[1] J. L. Shin, K. Tam, D. Huang, B. Petrick, H. Pham, C. Hwang, H. Li, A. Smith, T. Johnson, and F. Schumacher et al., "A $40 \mathrm{~nm}$ 16-core 128-thread CMT SPARC SoC processor," IEEE J. Solid-State Circuits, vol. 46, no. 1, pp. 131-144, Jan. 2011.

[2] H. Hatamkhani and R. Drost, "A 10-mW 3.6-Gbps I/O transmitter," in Symp. VLSI Circuits Dig. Tech. Papers, 2003, pp. 97-98.

[3] J. Poulton, R. Palmer, A. Fuller, T. Greer, J. Eyles, W. Dally, M. Horowitz, I. Rambus, and C. Hill, "A 14-mW 6.25-Gb/s transceiver in 90-nm CMOS," IEEE J. Solid-State Circuits, vol. 42, no. 12, pp. 2745-2757, Dec. 2007.

[4] K. Chang, H. Lee, J. Chun, T. Wu, T. J. Chin, K. Kaviani, J. Shen, X. Shi, W. Beyene, Y. Frans, B. Leibowitz, N. Nguyen, F. Quan, J. Zerbe, R. Perego, F. Assaderaghi, E. C. Real, and L. Altos, "A 16 Gb/s/link, 64 GB/s bidirectional asymmetric memory interface cell," in IEEE Symp. VLSI Circuits Dig., Jun. 2008, pp. 126-127.

[5] B. Kim, Y. Liu, T. O. Dickson, J. F. Bulzacchelli, and D. J. Friedman, "A 10-Gb/s compact low-power serial I/O with DFE-IIR equalization in 65-nm CMOS," IEEE J. Solid-State Circuits, vol. 44, no. 12, pp. 3526-3538, Dec. 2009.

[6] W. D. Dettloff, J. C. Eble, L. Luo, P. Kumar, F. Heaton, T. Stone, and B. Daly, "A $32 \mathrm{~mW} 7.4 \mathrm{~Gb} / \mathrm{s}$ protocol-agile source-series-terminated transmitter in $45 \mathrm{~nm}$ CMOS SOI," in IEEE Int. Solid-State Circuits Conf. Dig. Tech. Papers , 2010, pp. 370-371, IEEE.

[7] R. Sredojevic and V. Stojanović, "Digital link pre-emphasis with dynamic driver impedance modulation," in Proc. IEEE Custom Integrated Circuits Conf., San Jose, CA, Sep. 2010, pp. 1-4.

[8] V. Stojanovic and M. Horowitz, "Modeling and analysis of high-speed links," in Proc. IEEE Custom Integrated Circuits Conf., 2003, pp. 589-594.

[9] W. Peters, Q.-L. Chen, E. Gong, K. Cai, M. Austin, C. Chen, and Q. Chen, "ATCA Channel Data for Backplane Ethernet Task Force." [Online]. Available: www.ieee802.org/3/ap/public/channel_model

[10] H. Chung, A. Liu, and G. Y. Wei, "A 12.5-Gbps, 7-bit transmit DAC with 4-tap LUT-based equalization in $0.13 \mu \mathrm{m}$ CMOS," in Proc. IEEE Custom Integrated Circuits Conf., 2008, pp. 563-566.

[11] B. Kim and V. Stojanović, "An energy-efficient equalized transceiver for $R C$-dominant channels," IEEE J. Solid-State Circuits, vol. 45, no. 6, pp. 1186-1197, Jun. 2010.

[12] J. L. Zerbe, C. W. Werner, V. Stojanovic, F. Chen, J. Wei, G. Tsang, D. Kim, W. F. Stonecypher, A. Ho, T. P. Thrush, R. T. Kollipara, M. A. Horowitz, and K. S. Donnelly, "Equalization and clock recovery for a $2.5-10-\mathrm{Gb} / \mathrm{s}$ 2-PAM/4-PAM backplane transceiver cell," IEEE J. Solid-State Circuits, vol. 38, no. 12, pp. 2121-2130, Dec. 2003.

[13] T. H. Lee, The Design of CMOS Radio-Frequency Integrated Circuits. Cambridge, U.K.: Cambridge Univ., 2004.

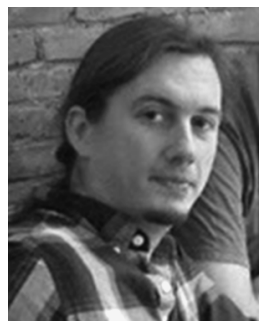

theory.
Ranko Sredojević (S'06) received the Dipl. Ing. degree in electronics from the School of Electrical Engineering, University of Belgrade, Belgrade, Serbia, in 2004, and the M.S. degree in electrical engineering from the Massachusetts Institute of Technology, Cambridge, in 2008, where he is currently working toward the Ph.D. degree in electrical engineering.

His current research interests include system simulation and optimization, circuits for high-performance communications and control, and control

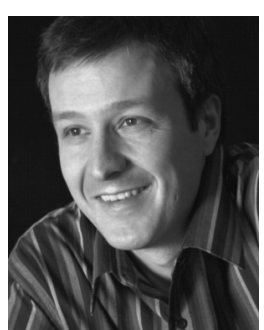

Vladimir Stojanović (S'96-M'05) received the M.S. and Ph.D. degrees in electrical engineering from Stanford University, Stanford, CA, in 2000 and 2005, respectively, and the Dipl. Ing. degree from the University of Belgrade, Belgrade, Serbia, in 1998.

$\mathrm{He}$ is currently the Emanuel E. Landsman Associate Professor with the Department of Electrical Engineering and Computer Science, Massachusetts Institute of Technology, Cambridge. He was with Rambus, Inc., Los Altos, CA, from 2001 to 2004. He was a Visiting Scholar with the Advanced Computer Systems Engineering Laboratory, Department of Electrical and Computer Engineering, University of California, Davis, during 1997-1998. His current research interests include design, modeling, and optimization of integrated systems, from novel switching and interconnect devices (such as NEM relays and silicon-photonics) to standard CMOS circuits.

Dr. Stojanovicć was a recipient of the 2009 National Science Foundation CAREER Award. 